\title{
A magnetic internal mechanism for precise orientation of the camera in wireless endoluminal applications
}

Authors

Institutions
P. Valdastri ${ }^{1}$, C. Quaglia' ${ }^{1}$ E. Buselli' , A. Arezzo ${ }^{2}$, N. Di Lorenzo ${ }^{3}$, M. Morino ${ }^{2}$, A. Menciassi ${ }^{1}$, P. Dario ${ }^{1}$

Center for Research in Microengineering (CRIM Lab), Scuola Superiore Sant'Anna, Pontedera, Italy

2 Centre for Minimally Invasive Surgery, University of Torino, Italy

3 Department of Surgery, University of Tor Vergata, Rome, Italy submitted 5 November 2009 accepted after revision 3 March 2010

\section{Bibliography}

DOI http://dx.doi.org/

$10.1055 / \mathrm{s}-0029-1244170$

Endoscopy 2010; 42:

481-486 @ Georg Thieme

Verlag KG Stuttgart · New York

ISSN 0013-726X

Corresponding author

\section{P. Valdastri, PhD}

CRIM Laboratory

Scuola Superiore Sant'Anna

viale R. Piaggio 34

56025 Pontedera

Italy

Fax: +39-0508-83497

pietro@sssup.it
Background and study aims: The use of magnetic fields to control operative devices has been recently described in endoluminal and transluminal surgical applications. The exponential decrease of magnetic field strength with distance has major implications for precision of the remote control. We aimed to assess the feasibility and functionality of a novel wireless miniaturized mechanism, based on magnetic forces, for precise orientation of the camera.

Materials and methods: A remotely controllable endoscopic capsule was developed as proof of concept. Two intracapsular moveable permanent magnets allow fine positioning, and an externally applied magnetic field permits gross movement and stabilization. Performance was assessed in ex vivo and in vivo bench tests, using porcine upper and lower gastrointestinal tracts.

Results: Fine control of capsule navigation and rotation was achieved in all tests with an external magnet held steadily about $15 \mathrm{~cm}$ from the capsule. The camera could be rotated in steps of $1.8^{\circ}$.

\section{Introduction}

$\nabla$

Although magnetic fields and their properties have been well known for centuries, their use in currently evolving surgical procedures is still limited, even if rapidly increasing. A promising field of application is the external magnetic control of diagnostic or surgical devices deployed inside the patient's body. In endoscopic procedures, this would allow maximization of the number of tools available with no effect on the number of access ports. In particular, magnetic camera systems can be introduced through natural orifices and oriented by magnetic forces.

Several solutions describing this approach have already been reported [1-5]. However, the static magnet on the endoscopic or surgical device always has a fixed orientation. Thus, the position and orientation of the device can only be con-
This was confirmed by ex vivo tests; the mechanism could adjust the capsule view at 40 different locations in a gastrointestinal tract phantom model. Full $360^{\circ}$ viewing was possible in the gastric cavity, while the maximal steering in the colon was $45^{\circ}$ in total. In vivo, a similar performance was verified, where the mechanism was successfully operated every $5 \mathrm{~cm}$ for $40 \mathrm{~cm}$ in the colon, visually sweeping from side to side of the lumen; $360^{\circ}$ views were obtained in the gastric fundus and body, while antrally the luminal walls prevented full rotation.

Conclusions: We report the feasibility and effectiveness of the combined use of external static magnetic fields and internal actuation to move small permanent intracapsular magnets to achieve wirelessly controllable and precise camera steering. The concept is applicable to capsule endoscopy as to other instrumentation for laparoscopic, endoluminal, or transluminal procedures.

trolled by varying the external magnetic field. In practice, this is obtained either by moving the external magnets, if permanent magnets have been used for the external platform, or by adjusting the magnetic field strength if coils have been used to generate the magnetic field. This results in poor and difficult control of the remote device because of the exponential decrease of magnetic field strength with distance. Control is further affected by the unpredictable presence of different tissues in between the external and the internal magnetic sources, depending on the position of the device inside the human body. Finally, because of the reliance on purely magnetic control, the remote device is maneuvered with respect to an entirely external reference frame. In endoluminal cavities, this usually means that the surrounding collapsed tissues move with the device when the external magnetic forces are applied; adjustabil- 

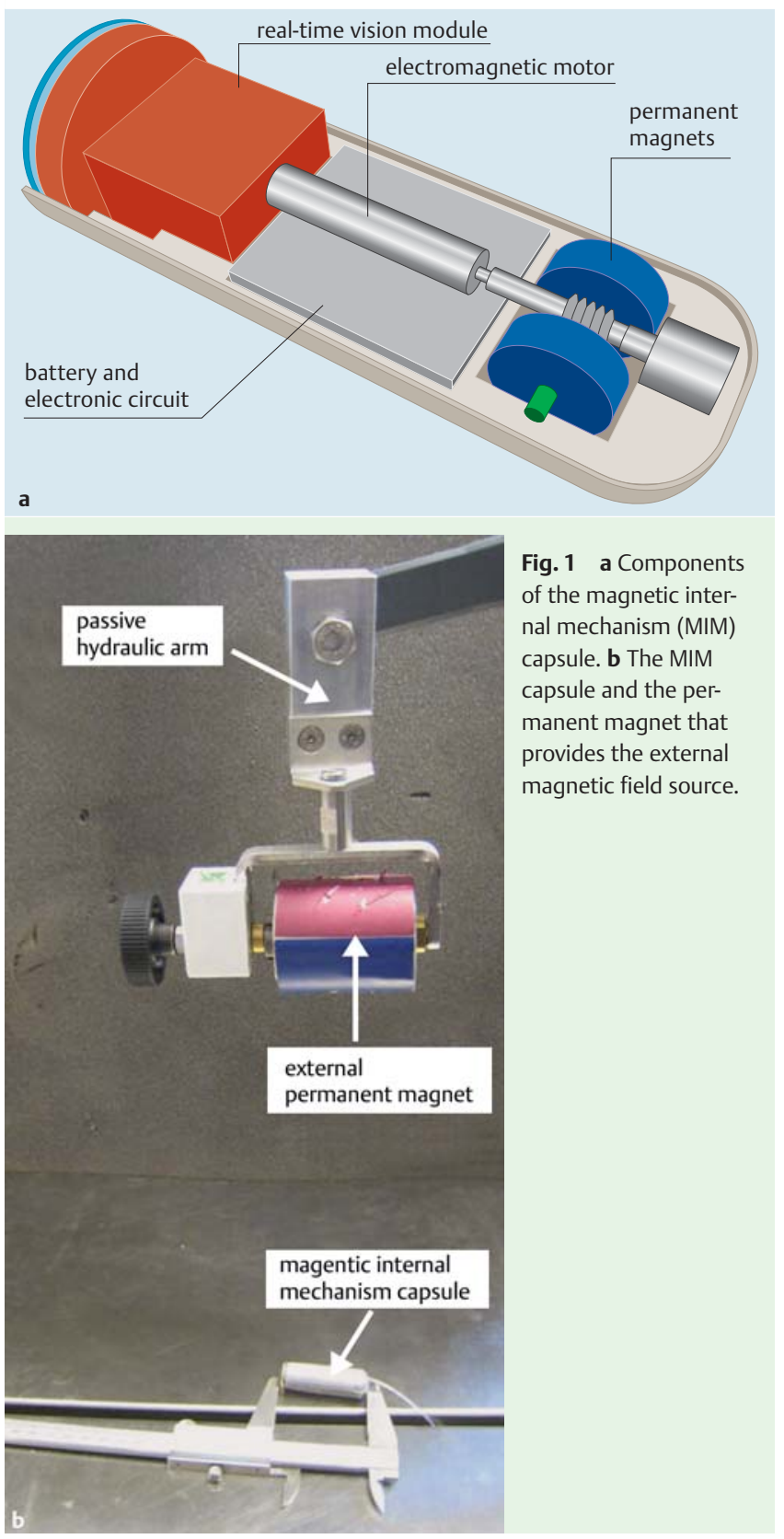

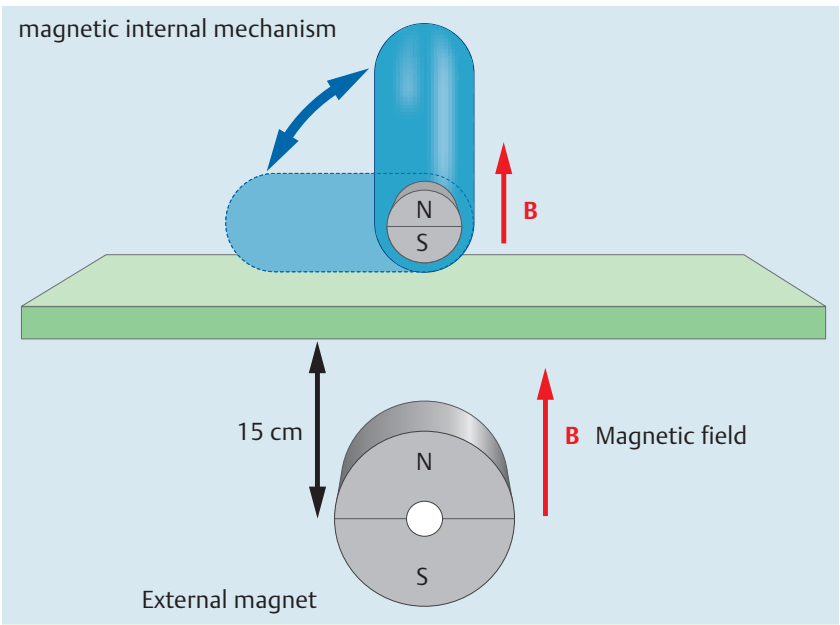

Fig. 2 The working principle of the magnetic internal mechanism (MIM) capsule. When the motor is activated, the capsule rotates as the intracapsular magnets stay in the same position with respect to the external magnet.

\section{Materials and methods}

The MIM capsule body ( Fig. $\mathbf{1}$ a) is cylindrical, with a diameter of $15 \mathrm{~mm}$ and a length of $48 \mathrm{~mm}$, and it incorporates one electromagnetic direct current brushless motor (Namiki Precision Jewel Co., Tokyo, Japan), two small permanent magnets diametrically magnetized (N52 NdFeB, Supermagnete, Uster, Switzerland), a rechargeable battery (3.7 V, 20 mAh, Plantraco, Saskatoon, Canada) and an electronic circuit for wireless motor control. A wired complementary metal-oxide-semiconductor (CMOS) camera (MO-S588-3T-N, Misumi, Taipei, Taiwan) with four white lightemitting diodes (LEDs) was used for the study because at present there is no wireless real-time compact camera on the market. Only the vision submodule is powered through the wired connection; the intracapsular battery provides energy for the wireless motor and controlling mechanism. The external lens has a hydrophobic coating to prevent moisture accumulation. The overall capsule weight is $14.4 \mathrm{~g}$.

The external magnetic field is provided by a cylindrical permanent magnet (N35 NdFeB, B\&W Technology \& Trade, China) ( $\bullet$ Fig. 1 b). It is $60 \mathrm{~mm}$ in diameter, $70 \mathrm{~mm}$ in length and $1.5 \mathrm{~kg}$ in weight, and it is magnetized diametrically. The external magnet is fixed in a passive hydraulic arm, which is manually controlled by the medical operator, similarly to an ultrasound device. A braking system allows the arm to be fixed in a given position by the user. The magnetic attraction force between the external and the internal magnets at a distance of $15 \mathrm{~cm}$ is $0.5 \mathrm{~N}$, roughly corresponding to $50 \mathrm{~g}$, which is enough to lift the MIM capsule against gravity.

Control software, running on a personal computer (PC), allows remote control of the device and feedback on its status, for example regarding battery level, wireless signal strength, etc. The user can rotate the camera view stepwise by pressing buttons on the PC keyboard, one for forward and one for backward motion. Alternatively, the rotation angle and direction can be set using the control software and an appropriate command is issued to the camera device.

The internal orienting mechanism consists of a motor connected to a worm gear. When the worm screw rotates, it transmits the motion to a toothed gear. The small intracapsular magnets are fixed to the toothed gear and rotate when the motor is activated. 
However, when the system is placed in an external magnetic field, the magnets tend to maintain a precise alignment with respect to the external magnet, based on the position of their own north and south poles. If the magnetic force is enough to withstand the torque of the motor, then the motor and the entire capsule rotates rather than the internal magnets which keep their orientation with regard to the external magnet ( $\bullet$ Fig. 2 ). This enables a fine adjustment of the camera relative to the surrounding tissue, without moving the external magnetic source. - Video 1 shows an example of operation of the MIM capsule during bench testing, where the internal mechanism rotates the capsule back and forth by $90^{\circ}$.

\section{Lower and upper gastrointestinal phantom models}

The main goal of ex vivo tests was to assess the reliability and precision of movement provided by the MIM once the capsule had been navigated magnetically to the target location. Quantitative outcomes were the minimum angular increments through which the camera could be rotated, the maximum steering angle that could be obtained in the colon and in the gastric cavity, and the possibility of achieving a $360^{\circ}$ sweep in the stomach.

An upper gastrointestinal model with fresh porcine esophagus and stomach inserts, and a lower gastrointestinal model ( $\bullet$ Fig. 3a) with fresh porcine colon inserts attached along the fixtures, were used to simulate ex vivo the anatomical geometry of the digestive tract. Four pairs of red and black targets were stitched in random positions along the upper digestive tract, and the same number of targets was used for the lower gastrointestinal experiment. Each red target was placed on the opposite side of the lumen to the corresponding black one. The external permanent magnet was fixed to a hydraulic passive arm in order to facilitate handling by the operator ( $\bullet$ Fig. $\mathbf{3 b}$ ).

The capsule was inserted through either the mouth or the anus of the phantom and it was guided by means of the external magnet to visualize the targets. A constant endoluminal slight insufflation, adequate for distension of the digestive tract, was applied. After each pair of targets had been approached, the external magnet was kept in a fixed position and the internal mechanism was activated through the control software in order to precisely steer the field of view from the black to the red target and back again. Two sessions, one for the colon and one for the stomach, were carried out, with five trials for each target, for a total of $40 \mathrm{MIM}$ activations.

\section{In vivo animal model}

After completion of the phantom trials, the MIM was assessed in an in vivo experimental session, with the aim of verifying the vision enhancement capabilities of the adjustable mechanism. The outcomes were confirmation of the capability of the MIM to move the view from one side to the other in the colonic lumen and to achieve a $360^{\circ}$ panoramic view at three different locations in the gastric cavity.

\section{Video 1}

Operation of the magnetic internal mechanism (MIM). When the intracapsular motor is activated, the entire capsule rotates as the intracapsular magnets retain their alignment with the external magnetic field.

online content including video sequences viewable at: www.thieme-connect.de/ejournals/abstract/endoscopy/ doi/10.1055/s-0029-1244170
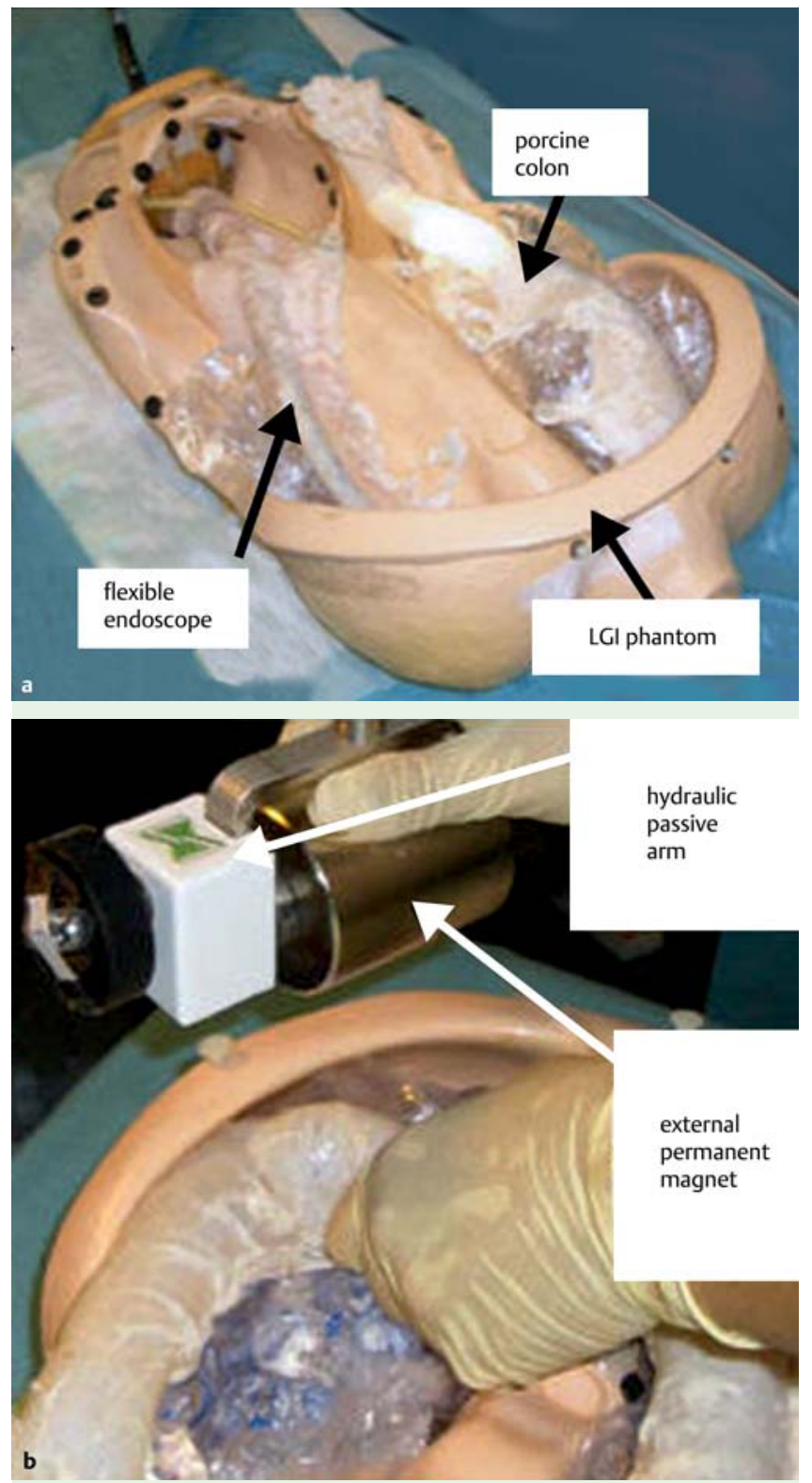

Fig. 3 a The lower gastrointestinal tract phantom set-up. b The phantom set-up and the external magnet on the hydraulic arm.

The feasibility study was done using two $30-\mathrm{kg}$ female domestic pigs. The experiments were carried out in an authorized laboratory, with the assistance and collaboration of a specially trained medical team, in accordance with all ethical considerations and the regulations related to animal experiments.

After intravenous sedation and bowel preparation using water enemas, the capsule device was inserted into the colon through the anus and advanced for $40 \mathrm{~cm}$ using external magnetic guidance. The advance was observed frontally by means of a flexible endoscope (Karl Storz, Tuttlingen, Germany) introduced through a colotomy at about $50 \mathrm{~cm}$ from the anus and directed towards the device. This also allowed moderate distension of the bowel. During the advance of the device, the intracapsular mechanism was activated every $5 \mathrm{~cm}$ in order to orient the vision module towards the lateral walls of the lumen and to acquire a detailed view of the mucosa. When the target location at $40 \mathrm{~cm}$ was reached, the capsule was moved magnetically backwards to the anus to be retrieved. 


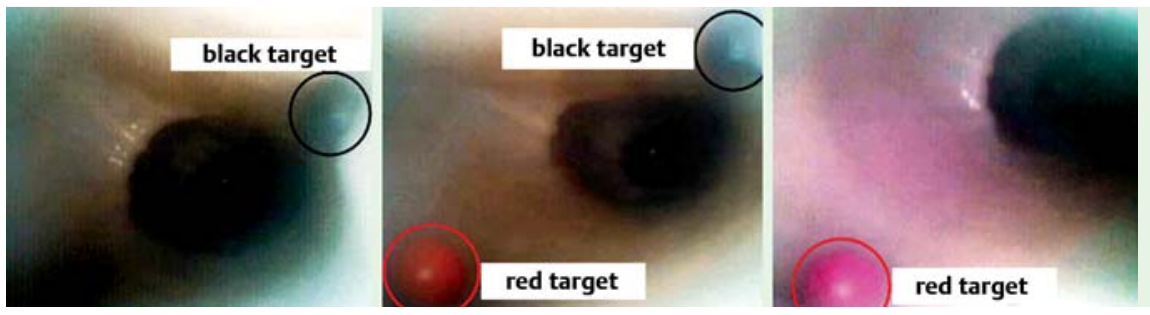

Fig. 4 View from the camera during lower gastrointestinal phantom trials.

Similarly the capsule was introduced into the stomach through a duodenotomy, while a flexible endoscope was inserted through the esophagus to achieve an optimal view and to provide sufficient insufflation. The capsule was then moved around the gastric cavity by external magnetic guidance and held in three different regions of the stomach, i.e., the fundus, the body, and the antrum. For each location, we tried to achieve a $360^{\circ}$ panoramic view by means of the intracapsular mechanism.

\section{Results \\ $\nabla$}

Lower and upper gastrointestinal phantom models

When each target area had been reached by gross magnetic navigation, the external magnet was fixed at $15 \mathrm{~cm}$ away the capsule. In these conditions, the MIM capsule was able to scan, moving from the black to the red target ( Fig. 4) and vice versa, in minimum angular rotation steps of $1.8^{\circ}$. A typical target located $20 \mathrm{~mm}$ from the camera was visualized in 2-mm increments. Regarding the maximum achievable sweep angle, a $360^{\circ}$ panoramic field of view was obtained in the phantom stomach, whereas in the colon the camera could be steered through no more than $45^{\circ}$ in total due to collision with the lateral walls.

Visualization and illumination, even if not comparable with that provided by standard flexible endoscopes, were sufficient for the sake of our tests.

The telemetric link between the PC and the capsule was reliable up to a distance of $3 \mathrm{~m}$. Battery lifetime, with continuous operation of the motor, was 25 minutes. The front lens did not need cleaning thanks to the hydrophobic coating of the glass.

\section{In vivo animal model}

Under in vivo conditions all the MIM functions performed correctly, including the telemetric motor commands. Continuous images were captured and the entire surface of both the stomach and colorectal tract along which the capsule was advanced were successfully visualized. Fine orientation was achieved with the external magnet fixed $15 \mathrm{~cm}$ away from the animal skin. In all eight MIM activations along the $40-\mathrm{cm}$ path in the colon, we were able to orient the camera from one side of the lumen to the other and back again, confirming the visualization in 2-mm steps. The trial in the colon was completed in 30 minutes and the battery was able to power the MIM for the entire duration.

\section{Video 2}

Endoscopic view of the magnetic internal mechanism (MIM) capsule rotating about a point during the in vivo experiment in the gastric cavity.

online content including video sequences viewable at: www.thieme-connect.de/ejournals/abstract/endoscopy/ doi/10.1055/s-0029-1244170

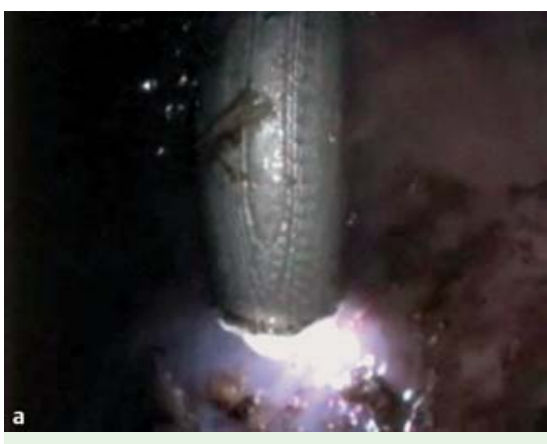

Fig. 5 Endoscopic view of three different positions of the magnetic internal mechanism (MIM) device, rotating about a point during the in vivo experiment in the gastric cavity.
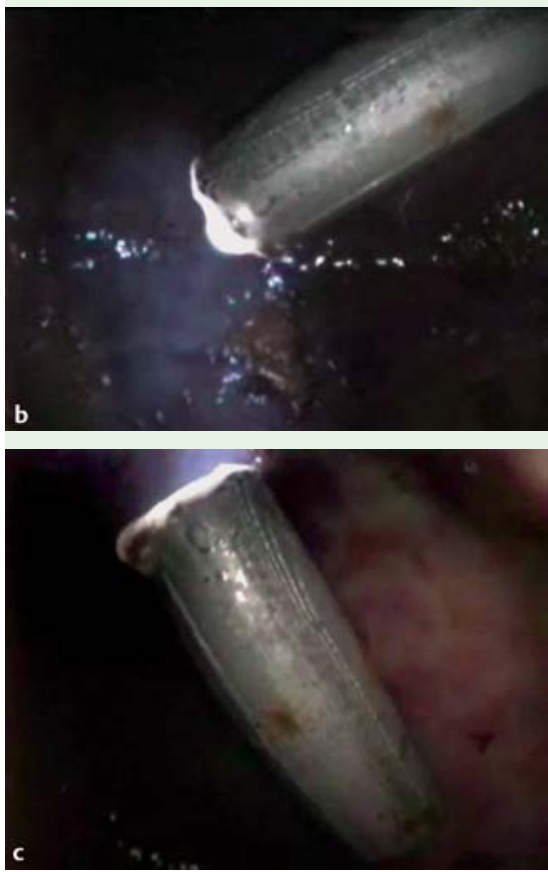

As regards the stomach trials, a 360-degree panoramic view ( Fig. 5a-c, Video 2) was achieved in the fundus and in the body, while in the antrum complete revolution was blocked by the gastric walls. An example of the image stream from the capsule is shown in $\bullet$ video 3 . The procedure time required to visualize the whole stomach surface was approximately 10 minutes. At the end of the entire trial, the capsule remained fully functional. Gross inspection of the animals after euthanasia revealed no mucosal damage.

\section{Video 3}

Video stream from the magnetic internal mechanism (MIM) on-board camera during the in vivo experiment in the gastric cavity.

online content including video sequences viewable at: www.thieme-connect.de/ejournals/abstract/endoscopy/ doi/10.1055/s-0029-1244170 


\section{Discussion}

\section{V}

In this study we introduce a novel concept for steering the video capsule camera, based on the combined use of an external static magnetic field and on-board actuation to move intracapsular permanent magnets. Since the distance between the external and internal magnets is kept constant during the operation, it is possible to achieve precise and reliable orientation of an endoluminal wireless device.

Transabdominal magnetic anchoring and guidance systems (MAGS) for minimally invasive surgery were first described in a laparoscopic setting by Cadeddu's group [1], and more recent application to natural orifice transluminal endoscopic surgery (NOTES) has been reported by the same group [2]. The use of magnetic fields has been tested under NOTES conditions by other groups to enable liver retraction during cholecystectomy as well as to stabilize a mesh for implantation during abdominal hernia repair [3]. Cuschieri's team used a ferromagnetic glue for gastric mucosa retraction in endoscopic surgery [4]. From the technical standpoint, the most advanced system to exploit magnetic fixation and positioning for laparoscopic and NOTES procedures is the peritoneum-mounted imaging robot as reported by Oleynikov's group [5]. This wired device contains a camera, an illumination system, an electromagnetic motor, and two permanent magnets located in fixed positions at the two ends of the device. A magnetic handle on the patient's abdomen is used to attract the magnets embedded in the robot. The handle can be moved across the exterior of the abdomen in order to position and pan the imaging device. The embedded motor is used to remotely tilt the camera to arbitrary angles. A similar solution is reported by Allen's group [6], consisting of a 11-mm monoscopic insertable panning/tilting endoscopic imaging device. A magnetically guided intra-abdominal camera for single-incision laparoscopic surgery (SILS) was recently demonstrated in humans by Cadeddu et al. [7].

Active capsule endoscopy is another field of research that is presently exploiting the combined use of external and intracapsular magnets. In this case magnetic fields are used to stop and control the capsule, and to steer it towards areas of interest for diagnostic $[8,9]$ and surgical [10] purposes. The efforts in this area by the two main commercial players in capsule endoscopy worldwide, namely Given Imaging [11] and Olympus [12], further attest to the promise of this approach.

The current trend in diagnostic and surgical procedures is to minimize the patient's operative trauma by decreasing as far as possible the number of external incisions while increasing the technical capabilities of endoluminal and transluminal instrumentation. Therefore, given the poor controllability of magnetic fields, the development of miniaturized mechanisms that allow precise positioning and steering of the single device would improve the outcomes of both standard and innovative procedures, such as laparoscopy, NOTES, SILS, or capsule endoscopy. We envisaged that magnetic properties could be used not only for retraction and for fixation of the intra-abdominal tools, but also for precise steering and rotation.

The novel feature of our prototype is the adjustable internal magnetic mechanism that allows fine control of steering and rotation. Compared with the steering achieved by moving the external magnetic field source, the mechanism presented here provides higher precision because the distance between the external and internal magnets is kept constant during the operation. As demonstrated by our findings, the best results with the MIM capsule are obtained in large cavities, such as the stomach or the abdomen, where movement of the device is not hampered by luminal walls. Nevertheless, even in a constrained environment, the MIM capsule's steering capabilities might be beneficial in enhancing diagnostic outcomes.

The MIM capsule design assumes a 15-cm distance between the external and internal magnets. With morbidly obese patients, such a value can easily be exceeded, and in such cases, a stronger external magnet can be used, by applying the selection criteria previously reported by our group [13].

It is worth mentioning that to be effective magnetic locomotion requires slight insufflation of the lumen [14]. In the case of gastric inspection, transparent liquid solutions can be used to achieve luminal distension [15]. Another promising approach using a wireless insufflation system for capsule endoscopy has been proposed [16]. Concerning power consumption, since the MIM is activated on demand by the user whenever steering of the camera is desirable, the precise battery lifetime cannot be predicted, but the user can take advantage of the battery level monitoring provided by the control software.

While feasibility for diagnostic evaluation of the upper and lower gastrointestinal tract was demonstrated, limitations to the development so far require comment. First, further reduction in size is desirable and this can be achieved by decreasing the size of the actuation unit. This would allow the diameter to be less than $12 \mathrm{~mm}$, thus enabling introduction through a standard trocar for abdominal operation. Further work on the visualization system are also needed to improve image quality. This is substandard in the current prototype; however the visualization system was not the main focus of this work. Integration of a wireless camera is also one of the next steps, in order to address capsule endoscopy application and to achieve a completely wireless device. This additional improvement will raise a significant issue regarding power consumption. A possible solution for supplying all the intracapsular subsystems within a reasonable volume may be wireless power induction [17]. On the other hand, use of the MIM capsule for laparoscopy, NOTES or SILS [13] would allow the presence of a tether. This wired connection could be used to power the system and to control both the camera and the MIM subsystems, thus solving any problem related to power consumption and allowing a drastic reduction in size, since battery and wireless electronics would not be required anymore.

Another crucial issue is the remote control by magnetic fields for navigation and coarse positioning. This problem may be addressed by adopting a robotic solution $[14,18,19]$.

Regarding the study protocol, the evaluation was done in ex vivo and in vivo nonsurviving porcine models. While we believe that all areas of the tracts explored were visualized, this was not definitively proven. In addition, the study was not designed to distinguish normal from abnormal mucosal findings. The obvious constraint was the specific anatomy of the porcine colon, that forced us to restrict the test to the distal area. Nevertheless we believe that when the downscaled version of our prototype might be available and a better external fine-control system developed and tested, further in vivo tests to prove feasibility and utility of a similar tool might be of interest, in view of the continuing development of evermore active and less invasive technologies for diagnosis and cure of digestive tract disease. 


\section{Acknowledgments}

$\nabla$

The authors are grateful to Mr. N. Funaro for manufacturing the prototypes and to Ekawahyu Susilo, Gastone Ciuti, and Carmen Cavallotti for their valuable support during fabrication and testing of the device.

This material is based in part upon work supported by the European Commission in the framework of the VECTOR (EU/IST2006-033970) and ARAKNES (EU/IST-2008-224-565) European Projects.

\section{Competing interests: None}

\section{References}

1 Park S, Bergs RA, Eberhart $R$ et al. Trocar-less instrumentation for laparoscopy magnetic positioning of intra-abdominal camera and retractor. Ann Surg 2007; 245: 379- 384

2 Scott DJ, Tang SJ, Fernandez R et al. Completely transvaginal NOTES cholecystectomy using magnetically anchored instruments. Surg Endosc 2007; 21: $2308-2316$

3 Ryou M, Thompson CC. Magnetic retraction in natural orifice transluminal endoscopic surgery (NOTES): addressing the problem of traction and countertraction. Endoscopy 2009; 41: $143-148$

4 Wang $Z$, Wang $L$, Tang $B$ et al. Retraction by surface ferromagnetisation of target tissues: preliminary studies on feasibility of magnetic retraction for endoscopic surgery. Surg Endosc 2008; 22: 1838-1844

5 Lehman AC, Berg KA, Dumpert J et al. Surgery with cooperative robots. Comput Aided Surg 2008; 13: 95-105

6 Hogle NJ, Hu T, Allen PK, Fowler DL. Comparison of monoscopic insertable, remotely controlled imaging device with a standard laparoscope in a porcine model. Surg Innov 2008; 15: 271-276
7 Cadeddu JA, Fernandez R, Desai M et al. Novel magnetically guided intra-abdominal camera to facilitate laparoendoscopic single-site surgery: initial human experience. Surg Endosc 2009; 23: 1894 - 1899

8 Carpi F, Galbiati G, Carpi A. Controlled navigation of endoscopic capsules: Concept and preliminary experimental investigations. IEEE Trans Biomed Eng 2007; 54: 2028-2036

9 Wang $X$, Meng $M Q H$. A magnetic stereo actuation mechanism for active capsule endoscope. Conf Proc IEEE Eng Med Biol Soc 2007; 2007: $2811-2814$

10 Valdastri P, Quaglia C, Susilo E et al. Wireless therapeutic endoscopic capsule. Endoscopy 2008; 40: 979-982

11 Volke F, Keller J, Schneider A et al. In-vivo remote manipulation of modified capsule endoscopes using an external magnetic field. Gastrointest Endosc 2008; 5: AB121 - AB122

12 Gheorghe C, Iacob R, Bancila I. Olympus capsule endoscopy for small bowel examination. J Gastrointest Liver Dis 2007; 16: 309-313

13 Simi M, Ciuti G, Tognarelli $S$ et al. Magnetic link design for a robotic laparoscopic camera. Journal of Applied Physics 2010; 107: 09B302

14 Ciuti G, Donlin R, Valdastri $P$ et al. Robotic versus manual control in magnetic steering of an endoscopic capsule. Endoscopy 2010; 42: $148-152$

15 Tortora G, Valdastri P, Susilo E et al. Propeller-based wireless device for active capsular endoscopy in the gastric district. Min Invas Ther Allied Technol 2009; 18: 280-290

16 Toennies JL, Webster RJ, III. A wireless insufflation system for capsular endoscopes. ASME Journal of Medical Devices 2009; 3: 27514

17 Carta $R$, Lenaerts B, Thoné $J$ et al. Wireless power supply as enabling technology towards active locomotion in capsular endoscopy. Biosens Bioelectron 2009; 25: 845 - 851

18 Ciuti G, Valdastri P, Menciassi A, Dario P. Robotic magnetic steering and locomotion of capsule endoscope for diagnostic and surgical endoluminal procedures. Robotica. 2010; 28: 199-207

19 Carpi F, Pappone C. Magnetic manoeuvring of endoscopic capsules by means of a robotic navigation system. IEEE Trans Biomed Eng 2009; 56: $1482-1490$ 